\title{
Mechanical Fault Diagnosis Method Based on LMD Shannon Entropy and Improved Fuzzy C-means Clustering
}

\author{
Shaojiang Dong \\ School of Automation, Chongqing University, Chongqing, 400044, Peoples Republic of China \\ School of Mechatronics and Automotive Engineering, Chongqing Jiaotong University, Chongqing 400074, Peoples \\ Republic of China
}

\section{Xiangyang Xu}

Chongqing University, College of Optoelectronic Engineering, 400044, Peoples Republic of China School of Mechatronics and Automotive Engineering, Chongqing Jiaotong University, Chongqing 400074, Peoples Republic of China

\begin{abstract}
Jiayuan Luo
School of Mechatronics and Automotive Engineering, Chongqing Jiaotong University, Chongqing 400074, Peoples Republic of China
\end{abstract}

(Received 31 August 2015; accepted 26 August 2016)

\begin{abstract}
Aiming to diagnosis the mechanical fault precisely, the current research is proposing a method based on the local mean decomposition (LMD) Shannon entropy and improved fuzzy f-means flustering (IFCM). The features are first extracted by using the time-frequency domain method LMD and the Shannon entropy is used to process the original separated product functions (PF) so as to extract the original features. However, the extracted original features are still with high dimensional and include superfluous information. The nonlinear multi-features fusion technique LTSA is used to merge the features and reduce the dimension. Then, based on the extracted features, the IFCM model is used to achieve the mechanical fault diagnosis. In this model, a feature cluster determine function is established to achieve the weighted factor to achieve a better category effect. Case of a bearing test was analyzed and the results proved the effectiveness of the methodology.
\end{abstract}

\section{INTRODUCTION}

Today, diagnosis is a very important research area in industry. Traditional concepts of preventive and corrective maintenance are gradually supplemented by diagnosis form. The main objective of this maintenance type is to ensure the dependability of industrial systems and increase their availability with a lower cost. However, fault diagnosis is not an easy task since it is essentially a problem of pattern recognition. A more effective feature extraction and accurate classifier are needed to obtain a higher diagnostic accuracy. ${ }^{1}$

Feature extraction is the process of transforming the raw vibration data collected from running equipment to relevant information of machine working condition. There are three types of methods that deal with the raw vibration data: time domain analysis, frequency domain analysis, and time-frequency domain analysis. These three methods are often chosen to extract a feature. For example, $\mathrm{Fan}^{2}$ chose the time-frequency domain transform ensemble empirical mode decomposition to describe the characteristics of the vibration signals. Qin $^{3}$ chose the wavelet transform to extract the feature of machinery wear information. The frequency features from FFT analysis results often tend to average out transient vibrations and thus do not provide a wholesome measure of the bearing health status. Hence, the time-frequency method is used rather frequently. Wavelet transformation is the most commonly used time-frequency analysis method and is widely used in feature extraction. ${ }^{4}$ However, the selection of the wavelet mother coefficient is difficult, and for different researchers the selected coefficient is not unique. For this reason, it is necessary to find an effective method to extract the fault-related features hidden in the complex and non-linear bearing vibration signals. The EMD ${ }^{5}$ is other tool usually used in time-frequency domain analysis which is suitable for analyzing non-stationary signals. However, the EMD method has the problems of endpoint leak, and the modal aliasing. In this article, the local mean decomposition (LMD) is used to processing the vibration signals, ${ }^{6}$ and the LMD Shannon entropy is used to extract the original features from the signal.

Although the original features can be extracted, they are still high dimensional and include superfluous information, which is why the original features fusion and dimensional reduction method should be used to deal with the original features so as to select the typical features. The most commonly used features fusion and dimensional reduction method is principal component analysis (PCA). ${ }^{7}$ However, the PCA is mainly used 
for dealing with the linear data set while the bearing vibration features are usually suppressed by the nonlinear characteristic features so the PCA cannot work effectively. Therefore, it is a challenge to find an effective nonlinear features fusion and dimensional reduction method. In this research, a new feature extraction method local tangent space alignment (LTSA) ${ }^{8}$ is chosen. The LTSA is an efficient manifold-learning algorithm, which can be used as a preprocessing method to transform the high dimensional data into more easily handled low dimensional data. This method has been used in many fields. In this paper, the LTSA is used to achieve extracting the more sensitive features.

Recognizing condition, in order to identify the work condition of machinery further, the SVM and BPNN models are typical used model served as a classifier. ${ }^{9}$ However, the SVM is not sensitive to the nonlinear feature classification. The BPNN model also carries some disadvantages i.e., the slow rate of learning and getting trapped in local minima. The FCM method based on learning analogy, it is very effective for statistical pattern recognition, and can achieve higher classification accuracy for unknown and non-normal distribution data. ${ }^{10,11}$ In this research, the FCM method is revised in order to select the features in order to reduce the classifier complex and computationally intensive, so we need to compress the features and supervise them so as to reduce the recognize error rate. Towards this end, a feature cluster determination function is established to achieve a better category effect.

\section{METHODS OF SIGNAL PROCESSING AND DIMENSIONAL REDUCTION}

\subsection{Original Vibration Process}

The nature of LMD is to demodulate AM-FM signals. By using LMD, a complicated signal can be decomposed into a set of product functions, each of which is the product of an envelope signal and a pure frequency-modulated signal. Furthermore, the completed time-frequency distribution of the original signal can be derived. For any signal $x(t)$, it can be decomposed as follows: ${ }^{6}$

(1) Determine all the local extreme points $n_{i}$ (including the maxima and minima) of the non-stationary signal, then calculate the mean $m_{i}$ of the two adjacent extreme pointsand $n_{i}$ and $n_{i+1}$, as well as the envelope estimation function $a_{i}$ :

$$
\begin{aligned}
& m_{i}=\frac{n_{i}+n_{i+1}}{2} ; \\
& a_{i}=\frac{\left|n_{i}-n_{i+1}\right|}{2} .
\end{aligned}
$$

Use straight lines to connect the two points adjacent to each mean $m_{i}$ and envelope the estimate $a_{i}$, respectively. Next, use the moving average method to obtain the local mean function $m_{11}(t)$ and envelope the estimation function $a_{11}(t)$.

(2) Separate the local mean function $m_{11}(t)$ from the signal $x(t)$ to obtain $h_{11}(t)$ :

$$
h_{11}(t)=x(t) m_{11}(t) .
$$

To de-modulate $h_{11}(t)$, divide it by the envelope estimation function $a_{11}(t)$ to obtain $s_{11}(t)$ :

$$
s_{11}(t)=\frac{h_{11}(t)}{a_{11}(t)} .
$$

(3) Ideally, $s_{11}(t)$ should be a pure frequency-modulated (FM) signal. However, this condition is not always fully satisfied in reality. Hence, it is necessary to treat $s_{11}(t)$ as the source data and repeat the above process iteratively until $s_{1 n}(t)$ is a pure FM signal. Considering the effects of decomposition, the number of iterations, speed, and other factors, the selected conditions for the termination of the iteration process are given by $a_{1 n}(t)=1$. When $s_{1 n}(t)$ satisfied the condition of being a pure FM signal, all the envelope estimation functions generated during the iterative process were multiplied to derive the envelope signal $a_{1}(t)$ (i.e., the instantaneous amplitude of the function) of the first $\mathrm{PF}$ component $P F_{1}(t)$ :

$$
a_{1}(t)=a_{11}(t) a_{12}(t) \cdots a_{1 n}(t)=\prod_{q=1}^{n} a_{1 q}(t)
$$

where $q$ represents the number of iteration loops. The single component of the AM-FM signal $P F_{1}(t)$ is given by:

$$
P F_{1}(t)=a_{1}(t) s_{1 n}(t)
$$

(4) The first PF component $P F_{1}(t)$ is separated from the signal $x(t)$ to obtain a new signal $u_{i}(t)$. Repeat the above step using as the source data for $k$ cycles until $u_{k}(t)$ becomes a monotonic function. In this way, $x(t)$ can be decomposed into the sum of $k$ PF components and $u_{k}(t)$ :

$$
x(t)=\sum_{p=1}^{k} P F_{p}(t)+u_{k}(t) .
$$

The use of Eq. (7) ensures that, following LMD, data from the original signal are better retained and is less likely to get lost.

\subsection{Feature Extraction}

Once the $k$ PFs and a residue $u_{k}(t)$ are obtained, where the energy of the $k$ PFs is $E_{1} ; E_{2} ; \ldots ; E_{k}$ can be calculated respectively then, due to the orthogonality of the LMD decomposition, the sum of the energy of the $k$ PFs should be equal to the total energy of the original signal when the residue $u_{k}(t)$ is ignored. As the PFs $c_{1}(t) ; c_{2}(t) ; \ldots ; c_{k}(t)$ include different frequency components, $\mathbf{E}=\left\{E_{1}, E_{2}, \ldots, E_{k}\right\}$ forms an energy distribution in the frequency domain of roller bearing vibration signal, and then the corresponding LMD energy entropy is designated as:

$$
H_{\text {entropy }}(t)=-\sum_{i=1}^{k} p_{i} \log p_{i}
$$

where $p_{i}=E_{i} / E$ is the percent of the energy of $c_{i}(t)$ in the whole signal energy $\left(\mathbf{E}=\sum_{i=1}^{k} E_{i}\right)$. 


\subsection{Basic Concepts of Local Tangent Space Alignment}

The basic idea of LTSA is to use the tangent space of sample points to represent the geometry of the local character. Then these local manifold structures of space are lined up to construct the global coordinates. Given a data set $X=$ $\left[x_{1}, x_{2}, \ldots, x_{k}\right], x_{i} \in R^{m}$, a mainstream shape of $d$-dimension $(m>d)$ is extracted. The LTSA feature extraction algorithm is as follows: ${ }^{8}$

1) Extract local information: for each $x_{i}, i=1,2, \ldots, k$, used the Euclidean distance to determine a set $x_{i}=$ $\left[x_{i, 1}, x_{i, 2}, \ldots, x_{i, f_{i}}\right]$ of its neighborhood adjacent points (e.g. $f$, nearest neighbors).

2) Local linear fitting: In the neighborhood of data points $x_{i}$, a set of orthogonal basis $Q_{i}$ can be selected to construct the $d$-dimension neighborhood space of $x_{i}$ and the orthogonal projection of each point $x_{i, j}(j=1,2, \ldots, k)$ can be calculated to the tangent space of $\theta_{j}^{(i)}=Q_{i}^{T}\left(x_{i, j}-\bar{x}_{i}\right) . \bar{x}_{i}$ is the mean data for the neighborhood. The orthogonal projection in the tangent space of neighborhood data of $x_{i}$ is composed of local coordinate $\Theta_{i}=\left[\theta_{(i), 1}, \theta_{(i), 2}, \ldots, \theta_{(i), f_{i}}\right]$ that describes the most important information of the geometry of the $x_{i}$.

3) Global order of the Local coordinates: supposing the global coordinates of $x_{i}$ converted by the $\Theta_{i}$ is $T_{i}=$ $\left[t_{i 1}, t_{i 2}, \ldots, t_{i f_{i}}\right]$, then the error is:

$$
E_{i}=T_{i}\left[I-(1 / f) e e^{T)}\right]-L_{i} \Theta_{i}
$$

where the $I$ is the identity matrix; the $e$ is the unit vector; the $f$ is the points number of the neighborhood; and the $L_{i}$ is the transformation matrix. In order to minimize the error, the $T_{i}$ and $L_{i}$ should be found, then:

$$
\begin{gathered}
L_{i}=T_{i}\left(I-(1 / f) e e^{T)}\right) \Theta_{i}^{*} ; \\
E_{i}=T_{i}\left(I-(1 / f) e e^{T)}\right)\left(I-\Theta_{i}^{*} \Theta_{i}\right) ;
\end{gathered}
$$

where $\Theta_{i}^{*}$ is the Moor-Penrose generalized inverse of $\Theta_{i}$. Supposing the

$$
B=P W W^{T} P^{T} .
$$

Let $P=\left[P_{1}, P_{2}, \ldots, P_{k}\right] ; T P_{i}=T_{i} ; P_{i}$ is a selected matrix from $0-1$; and the $T$ are global coordinates, their weight matrix

$$
\begin{gathered}
W=\operatorname{diag}\left(W_{1}, W_{2}, \ldots, W_{k}\right) ; \\
W_{i}=\left(I-(1 / f) e e^{T)}\right)\left(I-\Theta_{i}^{*} \Theta_{i}\right) .
\end{gathered}
$$

The constraint is:

$$
T T^{T}=I_{d}
$$

4) Extract of the low-dimensional manifolds feature: Since $e$ the is the eigenvalue of matrix $B$, so the corresponding minimum eigenvectors matrix is composed of eigenvalue. From 2 to $d+1$ of matrix $B$ make of the $T$. $T$ is the global coordinate mapping in the Mainstream form of low-dimensional transformed from the non-linear high-dimensional data set of $X$.

\section{THE IMPROVED FUZZY C-MEANS CLUSTERING METHOD MODEL}

Traditional cluster analysis requires every point of the data set to be assigned into a cluster precisely, so we call it hard clustering. But in fact, most things exist ambiguity in the attribute, there are no explicit boundaries among the things, and no the nature of either-or. So the theory of fuzzy clustering is more suitable for the nature of things and it can a more objective reflection of reality. Currently, the fuzzy c-means clustering (FCM) algorithm is the most widely used. ${ }^{11}$

FCM partitions set of $n$ objects $X=\left(x_{1}, x_{2}, \ldots, x_{n}\right)$ into $K$ fuzzy clusters with $C=\left(c_{1}, c_{2}, \ldots, c_{n}\right)$ cluster centers. In fuzzy matrix $U=\left(u_{i j}\right), u_{i j}$ is the membership degree of $i^{t h}$ the object with the $j^{\text {th }}$ cluster. The characters of $u_{i j}$ are as follows

$$
u_{i j} \in[0,1] \quad i \in 1,2, \cdots, n, \quad j \in 1,2, \cdots, K
$$

$$
\begin{gathered}
\sum_{j=1}^{K} u_{i j}=1 \quad i \in 1,2, \cdots, n \\
0<\sum_{i=1}^{n} u_{i j}<n \quad j \in 1,2, \cdots, K .
\end{gathered}
$$

Update $u_{i j}$ according to Eq. (16):

$$
u_{i j}=\left\{\begin{array}{c}
{\left[\sum_{k=1}^{K} \frac{d_{i j}^{b-1}}{d_{i k}^{b-1}}\right]^{-1}, \quad d_{i k} \neq 0} \\
0, \quad d_{i k}=0(k=j) \\
1, \quad d_{i j}=0(k \neq 0)
\end{array}\right.
$$

where: $b>1$ is the fuzziness exponentand, and $c_{j}(j \in[1, K])$ is the clustering center. $d_{i j}=\left\|x_{i}-c_{j}\right\|$.

Update $c_{j}$ according to Eq. (17):

$$
c_{j}=\frac{\sum_{i=1}^{n} u_{i j}^{b} x_{i}}{\sum_{i=1}^{n} u_{i j}^{b}} .
$$

The objective function is the Eq. (18):

$$
J(U, C)=\sum_{i=1}^{n} \sum_{j=1}^{K} u_{i j}^{b} d_{i j}^{2}
$$

FCM minimizes the objective function when $U_{i j}$ meets the conditions. The $U$ obtained from the algorithm is a fuzzy partition matrix, and it corresponds to the fuzzy partition of $X$. We apply the method of maximum subjection principle to get the certainty of partition:

In the $j^{\text {th }}$ column of $U$, if $u_{i j 0}=\max _{1<j<K}\left(u_{i j}\right), x_{i}$ merges into $j_{0}$. It means $x_{i}$ has the maximum membership degree to the cluster $j_{0}$, so we merge it into $j_{0}$.

In this research, a determine function is established to select the input features, so as to enlarge the distance of the different category and reduce the distance within the category. 


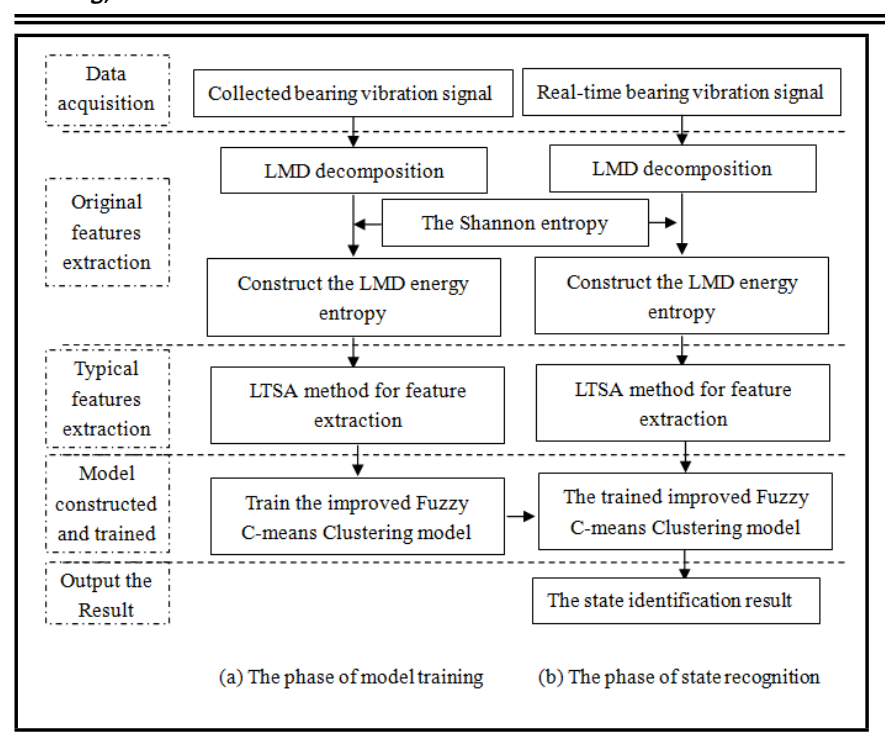

Figure 1. The flowchart of the proposed method.

Define the distance of the different category is $D_{a b}=$ $\frac{1}{N_{a} N_{b}} \sum_{i=1}^{N a} \sum_{i=1}^{N_{b}} D\left(x_{i}^{a}, x_{j}^{b}\right)$.

Define the distance of the same category is $D_{a}=$ $\min \left\|x_{i}-\bar{x}\right\|^{2}$.

So as the enlarge the distance the different category and reduce the distance of the same category, then the determine function is defined as:

$$
s=\frac{d_{a b}}{d_{a a}}=\frac{\frac{1}{N_{a} N_{b}} \sum_{i=1}^{N_{a}} \sum_{i=1}^{N_{b}} D\left(x_{i}^{a}, x_{j}^{b}\right)}{\min \left\|x_{i}-\bar{x}\right\|^{2}} ;
$$

where $D_{a}$ and $D_{b}$ represent the different category and $D_{a b}$ represents the distance of different category. $\bar{x}$ represents the average value of one category.

The weight ratio of all the different categories were calculated, and then the weight factor $\lambda_{i}=s_{i} / \sum_{i=1}^{c} s_{i}$ was used to improve Eq. (18)

$$
J(U, C)=\sum_{i=1}^{n} \sum_{j=1}^{K} \lambda_{i} u_{i j}^{b} d_{i j}^{2} .
$$

\section{PROPOSED METHOD}

The flowchart of the proposed method is shown in Fig. 1. The method consists of three procedures sequentially: data processing and features extraction, merge of the original features, constructing, and training IFCM model. The role of each procedure is explained as follows:

Step 1. Data processing and features extraction. The timefrequency domain signal processing method LMD is used to extract the original features from the collected mass vibration data.

Step 2. Merge of the original features. The LTSA method is used to extract the typical features and reduce the dimension of the features. The extracted features are used for training the FCM model.
Table 1. A group of LMD energy entropies of different running states of the actual signal.

\begin{tabular}{|c|c|c|c|c|c|}
\hline Running states & $H_{1}$ & $H_{2}$ & $H_{3}$ & $H_{4}$ & $H_{5}$ \\
\hline Normal state & 1.2657 & 1.2316 & 1.1091 & 1.1012 & 1.1316 \\
\hline 0.18 mm fault depth & 1.0684 & 1.0201 & 1.2452 & 1.2566 & 1.3910 \\
\hline $0.36 \mathrm{~mm}$ fault depth & 0.9506 & 0.9896 & 0.9597 & 1.1166 & 0.9259 \\
\hline $0.53 \mathrm{~mm}$ fault depth & 0.8923 & 0.8369 & 0.8949 & 0.8283 & 0.8675 \\
\hline $0.71 \mathrm{~mm}$ fault depth & 0.6031 & 0.5108 & 0.6392 & 0.6967 & 0.7042 \\
\hline
\end{tabular}

Step 3. Construct the FCM model. The determine function is used to improve the FCM. The IFCM model is used to fault diagnosis.

\section{VALIDATION AND APPLICATION}

\subsection{Case 1}

The proposed method was applied to bearing fault signals and the used data set in this paper is obtained from Case Western Reserve University. ${ }^{12}$ The bearing type in the experiments is SKF 6205-2RS JEM. Experiments were conducted by using a $2 \mathrm{hp} \mathrm{reliance} \mathrm{electric} \mathrm{motor.} \mathrm{Bearings} \mathrm{were} \mathrm{seeded} \mathrm{with} \mathrm{faults}$ by using electro-discharge machining. The test is to simulate the bearing normal running state and fault running states, with fault depths of $0.18 \mathrm{~mm}, 0.36 \mathrm{~mm}, 0.53 \mathrm{~mm}$, and $0.71 \mathrm{~mm}$ at the inner raceway, outer raceway and the ball to reflect the deteriorating state of the bearing; the outer- raceway fault signals were chosen in this case. Data was collected at the rate of 12,000 samples per second. Approximately 4096 data points were selected for analysis. There were 50 groups of test data for each fault states that were selected with 20 groups for training and the other 30 groups for testing.

The collected signal is first decomposed by LMD; each signal can be decomposed into a number of PF components (in this research, the number is 6), and then the first five PF components that may contain main fault information are selected to extract the features. The Shannon entropy was used to extract the features. The collected 4096 data points of $0.18 \mathrm{~mm}$ fault signal and its FFT result is shown in Fig. 2, the PFs (decomposed into 6 PFs ) decomposed by LMD as shown in Fig. 3:

The Shannon entropy is used to extract the features of the vibration signal. A group of Shannon entropy is obtained as shown in Table 1 (not normalized before).

Because there are 20 groups features for training and the groups of entropy values are still in high dimension, the LTSA method (the input data dimension is set to 3 , and the number of neighborhood is set to 12) is used to pre-process the entropy features; the result will work as the input of the improved FCM fault diagnosis model.

After the feature pre-process using the LTSA, the extracted features are inputted into the improved FCM model to train the model so as to recognize the states (the category number $k$ is set to 5). In order to compare the identifying effect of different methods, the following comparisons were done:

(1) Use LMD Shannon entropy to extract the features and directly input the extract features into the IFCM without the LTSA dimension reduction process. 


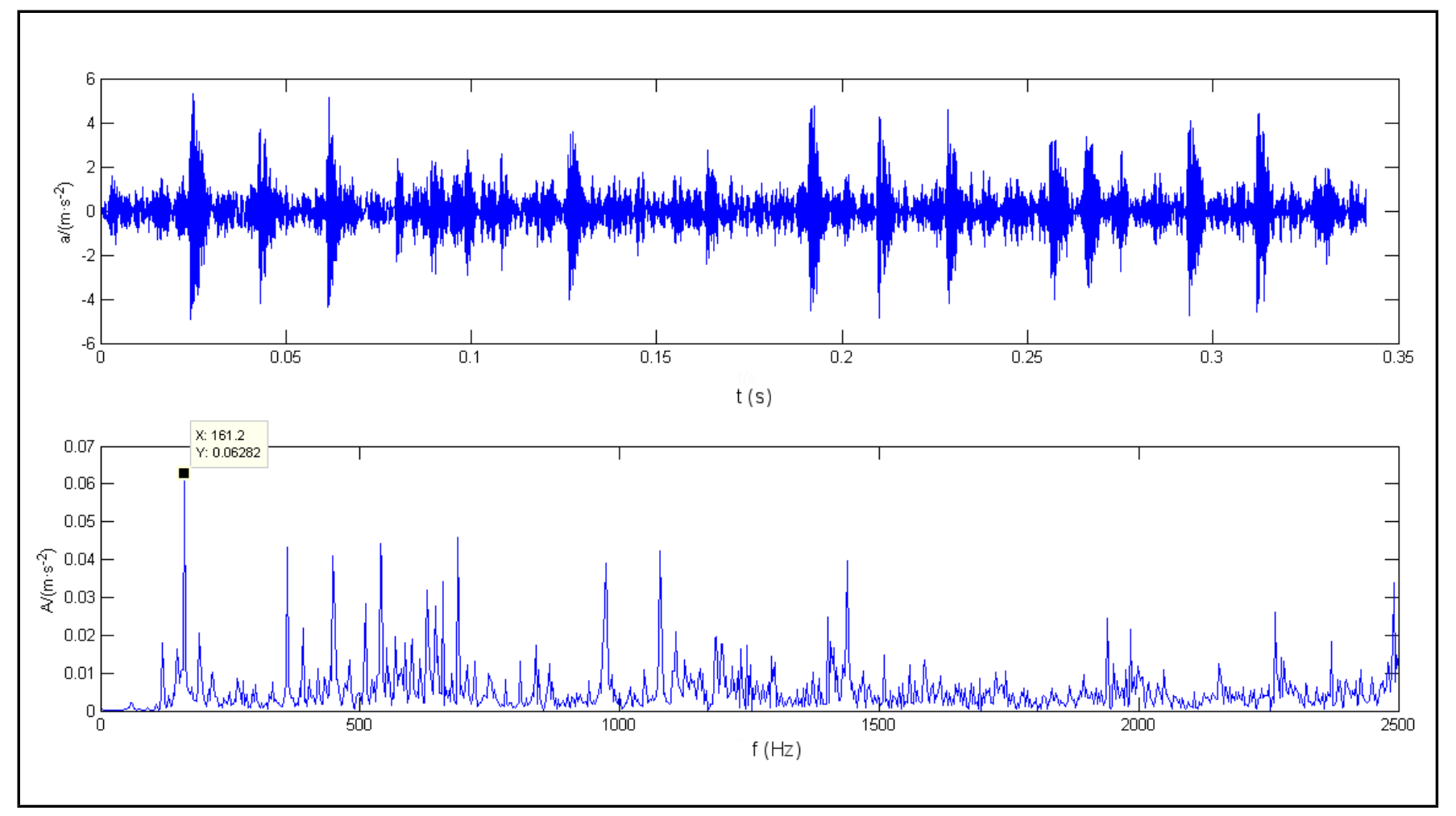

Figure 2. The collected 4096 data points of $0.18 \mathrm{~mm}$ fault signal and its FFT.

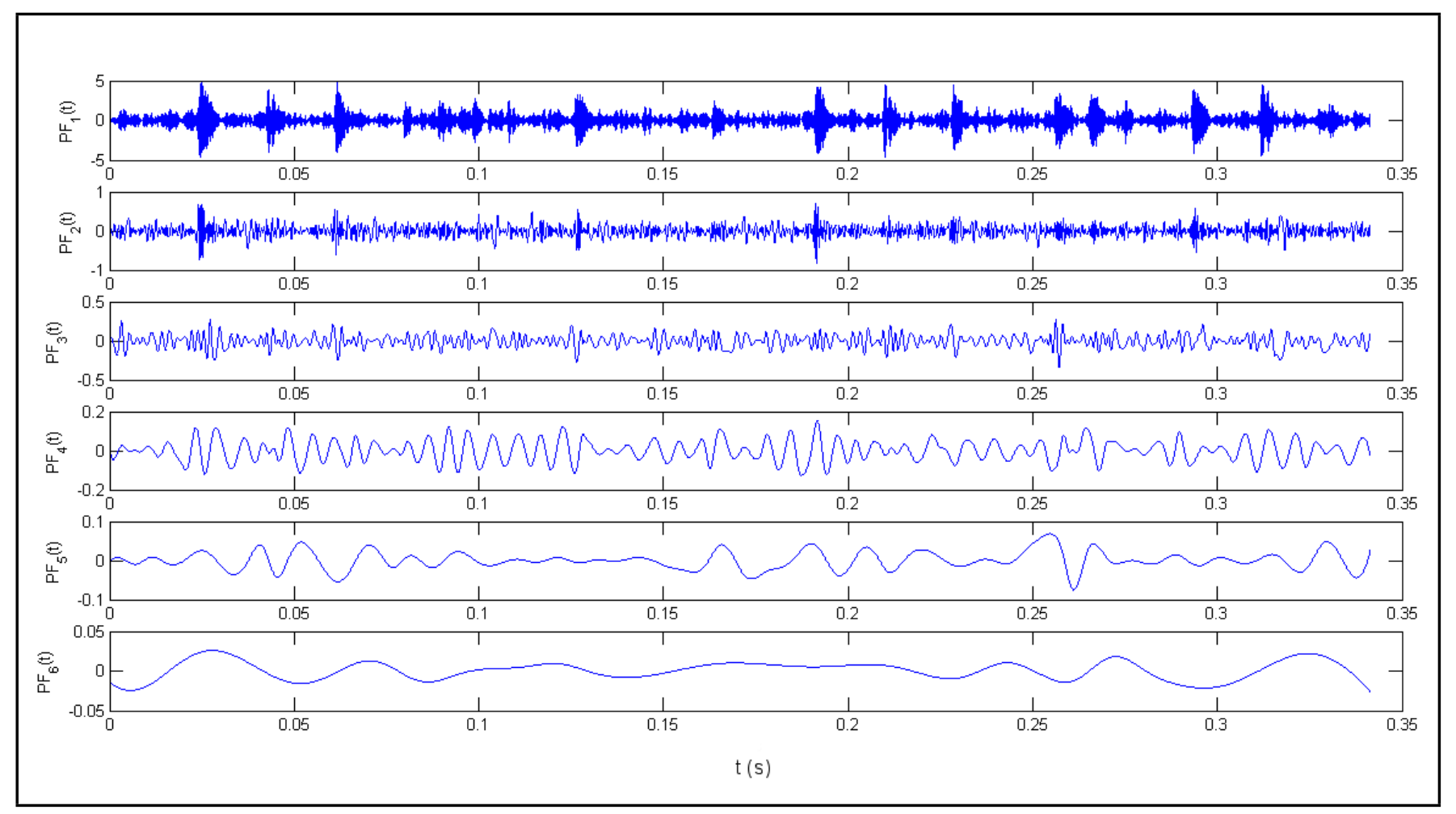

Figure 3. The PFs decomposed by the collected signal. 


Table 2. The recognition rate of different methods.
\begin{tabular}{|c|c|c|c|c|c||}
\hline \multirow{5}{*}{ Model type } & Recognition rate \% \\
\cline { 2 - 6 } & Normal & $\begin{array}{c}0.18 \\
\mathrm{~mm} \\
\text { fault } \\
\text { depth }\end{array}$ & $\begin{array}{c}0.36 \\
\mathrm{~mm} \\
\text { fault } \\
\text { depth }\end{array}$ & $\begin{array}{c}0.53 \\
\mathrm{~mm} \\
\text { fault } \\
\text { depth }\end{array}$ & $\begin{array}{c}0.71 \\
\mathrm{~mm} \\
\text { fault } \\
\text { depth }\end{array}$ \\
\hline $\begin{array}{c}\text { Without the LTSA } \\
\text { Pre-processed } \\
\text { by the PCA }\end{array}$ & 63 & 60 & 77 & 65 & 89 \\
\hline $\begin{array}{c}\text { Pre-processed } \\
\text { by the KPCA }\end{array}$ & 90 & 91 & 92 & 96 & 97 \\
\hline $\begin{array}{c}\text { The fault diagnosis } \\
\text { FCM model } \\
\text { that is not improved }\end{array}$ & 92 & 100 & 93 & 95 & 96 \\
\hline $\begin{array}{c}\text { The proposed } \\
\text { method }\end{array}$ & 100 & 100 & 100 & 100 & 100 \\
\hline
\end{tabular}

(2) Use LMD Shannon entropy to extract the features and process the extracted features by $\mathrm{PCA}^{7}$ to reduce the dimension, then input the features into the IFCM.

(3) Use LMD Shannon entropy to extract the features and process the extracted features by KPCA ${ }^{13}$ to reduce the dimension, then input the features into the IFCM.

(4) Use LMD Shannon entropy to extract the features and process the extracted features by LTSA to reduce the dimension, then input the features into the FCM.

(5) The method proposed in this article.

The comparison results are shown in Table 2. From the Table 2 it can be seen that method 1 omitted the process of using the LTSA to extract the features. Hence, the features with a high dimension and the IFCM model cannot deal with the features effectively; the resulting recognition rate is relatively low than other methods. Method 2 works without the LTSA by using the PCA and the results are inputted into the IFCM model, if the result is not good, the PCA model can achieve some fault identify such as the running state of normal and $0.71 \mathrm{~mm}$ seriously fault depth, however, the model cannot identify the bearing fault of other depths effectively, that is because the PCA model is not easy to deal with the nonlinear feature. Method 3 works without the LTSA by using the KPCA. The features extracted by the KPCA were inputted into the IFCM. The result was also not satisfactory because the KPCA model can preliminarily distinguish the features of different fault states, which can have a better effect than the PCA. However, in many states the KPCA is still not working very well for complex data, so the IFCM cannot deal with the features effectively. Method 4 proposed that the FCM is not improved; the results show some recognize error because the FCM method may fall into local recognize error when the data is complex. Hence, an improvement of the FCM model is required and the weight factor can help improve the recognize rate of the FCM. So the results of different methods proved the effect of the proposed method.

\subsection{Case 2}

The bearings are hosted on the shaft; the shaft is driven by AC motor. The rotation speed is kept at $1000 \mathrm{rpm}$ and a radial load of $3 \mathrm{~kg}$ is added to the bearing. The test rig is shown in Fig. 4. The data sampling rate is $25600 \mathrm{~Hz}$ and the data length is 102400 collected points, as shown in Fig. 5. Every two hours, the vibration data is collected. The bearing is run

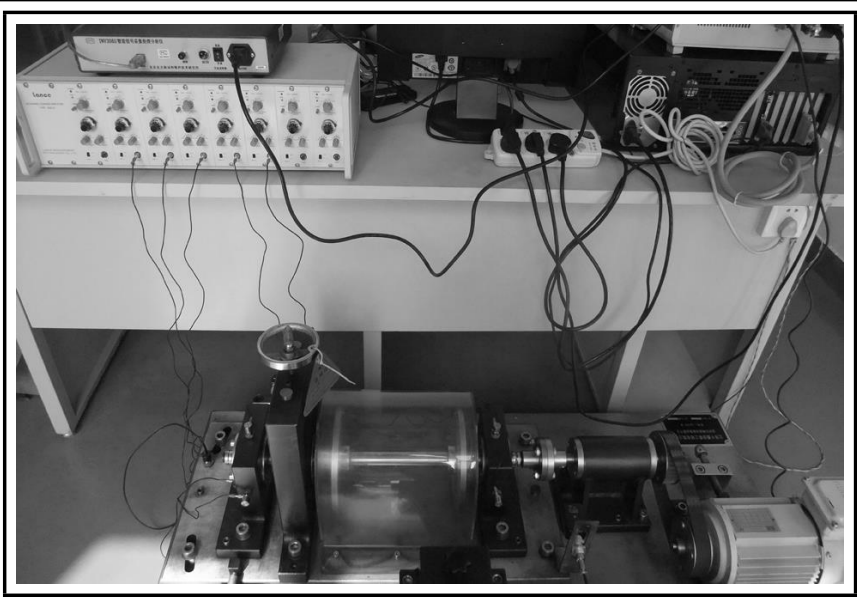

Figure 4. The test rig of Case 2.

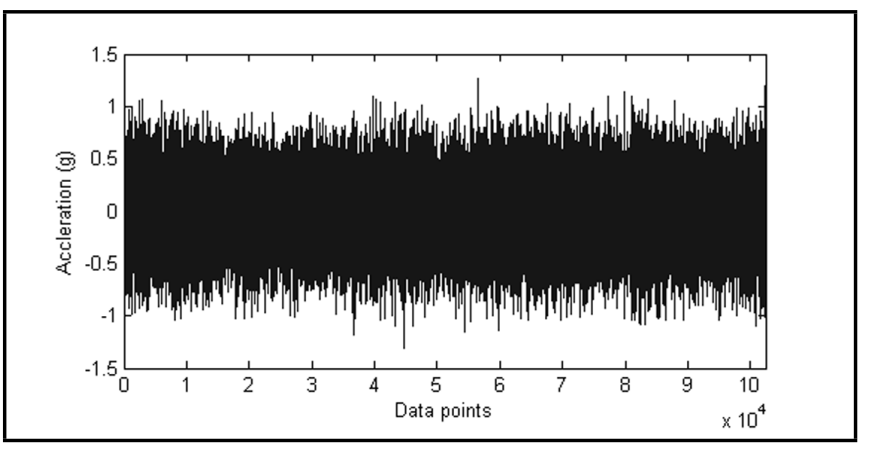

Figure 5. The collected vibration signal.

for one year. Then, a set of data from each of the two months is selected and the data sets are used to test whether or not the proposed method can identify the bearing running state. There were 8192 data points that were selected to be analyzed, 60 groups of collected data of different faults were obtained with 30 groups for training and the other 30 groups for testing. Next, the LMD method is used to decompose each group of signals, get the the PFs and the Shannon entropy of the calculated PFs. A group of features of different fault conditions are obtained, as shown in Table 3 (not normalized beforehand).

Then, the 30 groups' entropy values are normalized and input into the LTSA in order to pre-processe the typical featuresand the extracted features are input into the IFCM. The recognized results are shown in Table 4. Table 4 shows that the proposed method yields a high recognize accuracy even though the actual bearing running state is very complex. The running state of the normal state is about $95 \%$ accurate rate, which is because the bearing in running in safe state, the state is not clearly as the bearing running for 2 months, where the bear-

Table 3. A group of LMD energy entropies of different running states of the actual signal.

\begin{tabular}{|c|c|c|c|c|c|}
\hline Running states & $H_{1}$ & $H_{2}$ & $H_{3}$ & $H_{4}$ & $H_{5}$ \\
\hline Normal state & 1.2657 & 1.2316 & 1.1091 & 1.1012 & 1.1316 \\
\hline Running for 2 month & 1.0684 & 1.0201 & 1.2452 & 1.2566 & 1.3910 \\
\hline Running for 4 month & 0.9506 & 0.9896 & 0.9597 & 1.1166 & 0.9259 \\
\hline Running for 6 month & 0.8923 & 0.8369 & 0.8949 & 0.8283 & 0.8675 \\
\hline Running for 8 month & 0.6031 & 0.5108 & 0.6392 & 0.6967 & 0.7042 \\
\hline Running for 10 month & 0.7663 & 0.7095 & 0.7953 & 0.7325 & 0.8034 \\
\hline Running for 12 month & 0.7509 & 0.7993 & 0.8331 & 0.7408 & 0.8296 \\
\hline
\end{tabular}

International Journal of Acoustics and Vibration, Vol. 22, No. 2, 2017 
Table 4. The states of recognition rate of different states based on the proposed method (recognition rate $\eta \%$ ).

\begin{tabular}{||c|c|}
\hline Running states & recognition rate $\eta \%$ \\
\hline Normal state & 94 \\
\hline Running for 2 month & 97 \\
\hline Running for 4 month & 95 \\
\hline Running for 6 month & 96 \\
\hline Running for 8 month & 95 \\
\hline Running for 10 month & 100 \\
\hline Running for 12 month & 100 \\
\hline \hline
\end{tabular}

ing state was changing, the Shannon entropy were different with the normal state. The running state of 10 months and 12 months are recognized clearly, this is because the bearing state was degenerating. The results confirm that the proposed method can recognize the bearing running states effectively.

\section{CONCLUSIONS}

Firstly, this research used the LMD Shannon entropy method to extract the original features from the vibration signals. The LTSA was used to reduce the dimension and data redundancy of the entropy features. Using these methods, the typical features could be extracted effectively.

Then, in order to more accurately identify the bearing running state, the FCM model is improved and the weight factor is used so as to improve the recognition accuracy of FCM effectively.

Thirdly, through different comparisons we can see that the proposed method makes good use of the advantage of all parts and together to obtain better recognition accuracy and efficiency.

Finally, through the tested signals in the research, the results show the significant efficacy of the proposed method in identifying the bearing faults.

\section{ACKNOWLEDGEMENTS}

This research was supported by the National Natural Science Foundation of China (No. 51405047, 51405048), the Scientific and Technological Research Program of Chongqing Municipal Education CommissionNo.KJ1500529, KJ1500516), the China Postdoctoral Science Foundation (No.2016M590861), Chongqing Postdoctoral Science Foundation funded project (No. xm2015001, xm2015011), and the Natural Science Foundation Project of CQ cstc2015jcyjA70012. Natural Science Foundation Project of CQ cstc2014jcyjA1381.China Postdoctoral Science Foundation funded this research, Project no. 2014M552316. The authors are grateful to the anonymous reviewers for their helpful comments and constructive suggestions.

\section{REFERENCES}

1 Jaouher, B. A. and Nader, F. and Lotfi S. Application of empirical mode decomposition and artificial neural network for automatic bearing fault diagnosis based on vibration signals, Applied Acoustics, 89,16-27, (2015).
2 Fan, J. and Zhencai Z., and Wei L. Fault identification of rotor-bearing system based on ensemble empirical mode decomposition and self-zero space projection analysis, Journal of Sound and Vibration, 333, 3321-3331, (2014).

3 Yi, Q. and BaoPing T.and JiaXu W. Higher-density dyadic wavelet transform and its application, Mechanical Systems and Signal Processing, 24 , 823-834, (2010).

4 Shaojiang, D., Baoping, T., and Renxiang, C. Bearing running state recognition based on non-extensive wavelet feature scale entropy and support vector machine, Measurement, 46, 4189-4199, (2013) .

5 Baoping, T. and Shaojiang, D. and Tao S. Method for eliminating mode mixing of empirical mode decomposition based on the revised blind source separation, Signal Processing, 92 ,248-258, (2012).

6 Jiedi, S., Qiyang, X., and Jiangtao, W. Natural gas pipeline small leakage feature extraction and recognition based on LMD envelope spectrum entropy and SVM, Measurement, 55, 434-443, (2014).

7 Shaojiang, D. and Tianhong, L. Bearing degradation process prediction based on the PCA and optimized LS-SVM model, Measurement, 46, 3143-3152, (2013).

8 Shichang D. Minimal Euclidean distance chart based on support vector regression for monitoring mean shifts of auto-correlated processes, International Journal of Production Economics, 141, 377-387, (2013).

9 Yunluo, Y. and Wei, L. A novel sensor fault diagnosis method based on Modified Ensemble Empirical Mode Decomposition and Probabilistic Neural Network, Measurement, 68, 328-336, (2015) .

10 Telmo, M.,Silva, F., and Bruno, A. P., Hybrid methods for fuzzy clustering based on fuzzy c-means and improved particle swarm optimization Expert Systems with Applications, 42, 6315-6328, (2015).

11 Benaichouche, A. N., Oulhadj, H., and Siarry P. Improved spatial fuzzy c-means clustering for image segmentation using PSO initialization, Mahalanobis distance and postsegmentation correction Digital Signal Processing, 23, 1390-1400, (2013).

12 Case Western Reserve University bearing data center, 2009. Available from: http://www.eecs.cwru.edu/laboratory/bearing.

13 Qi W. Product demand forecasts using wavelet kernel support vector machine and particle swarm optimization in manufacture system, Journal of Computational and Applied Mathematics, 233, 2481-2491, (2010). 